\title{
Risk of developing dementia in people with diabetes and mild cognitive impairment
}

\author{
Latha Velayudhan, Michaela Poppe, Nicola Archer, Petroula Proitsi, Richard G. Brown
} and Simon Lovestone

\section{Background}

Diabetes mellitus is associated with cognitive dysfunction, but it is not clear whether the disorder increases the risk of conversion from mild cognitive impairment to dementia.

\begin{abstract}
Aims
To determine the association between diabetes mellitus and dementia conversion in people with mild cognitive impairment (Peterson's criteria) in a prospective communitybased study.

\section{Method}

People over 65 years old were approached through primary care practices in south London, UK, and those with mild cognitive impairment $(n=103)$ were followed up for 4 years Presence of diabetes was established from self-report and information from general practitioners.
\end{abstract}

\section{Results}

Nineteen participants progressed to dementia, with the predominant diagnosis being probable or possible Alzheimer's disease (in 84\%). Only diabetes mellitus was associated with progression to dementia (hazard ratio 2.9, $95 \% \mathrm{Cl} 1.1-7.3)$ after adjustment for sociodemographic factors, APOE4, premorbid IQ and other health conditions.

\section{Conclusions}

Diabetes mellitus increases not only the risks of dementia and mild cognitive impairment but also the risk of progression from such impairment to dementia.

\section{Declaration of interest}

None.
Dementia is one of the most common and most devastating diseases of late life; approximately 4.6 million new cases of dementia are estimated to occur worldwide every year and the number of people affected is predicted to double every 20 years to 81.1 million by $2040 .{ }^{1}$ People with mild cognitive impairment are at increased risk of developing dementia, although the conversion rates reported range from $1 \%$ to $25 \%$ or more per year. ${ }^{2}$ Prediction of progression to dementia - conversion - is of considerable clinical importance. To date there is no variable other than cognitive impairment itself that unequivocally increases the risk of conversion. Diabetes mellitus is associated with cognitive dysfunction. Type 2 diabetes mellitus has been related to accelerated cognitive decline in elderly people, development of mild cognitive impairment and increased risk of dementia, including both Alzheimer's disease and vascular dementia. ${ }^{3-7}$ With the global number of people with diabetes set to rise over the next few decades, ${ }^{8}$ the evidence that diabetes is a risk factor for dementia is of considerable public health importance. However, although the association between diabetes mellitus and risk of dementia is robust, it is not yet clear whether diabetes mellitus increases the risk of conversion from mild cognitive impairment to dementia. As early identification strategies are frequently targeted at people with mild impairment this is of clinical relevance - faced with a patient with mild cognitive impairment, the clinician needs to be able to judge the risk of conversion to dementia relative to the chances of the impairment remaining mild and causing no functional difficulty. We aimed to determine the association between diabetes mellitus and conversion to dementia in individuals with mild cognitive impairment over a follow-up period of 4 years in a prospective community-based study.

\section{Method}

White European men and women aged 65 years or above with no psychiatric or neurological disorder were recruited as part of a longitudinal cohort study. As previously described, ${ }^{9,10}$ study participants with no dementia were recruited through local primary care practices in south London. Potential candidates identified from the general practice registers were contacted by post and invited to participate. Those who responded positively were assessed either in their own home or at the practice surgery. Following baseline assessment, participants were assessed annually from 2001 through 2007.

\section{Diagnosis of cognitive impairment}

Cognitive assessments at baseline were conducted with the MiniMental State Examination (MMSE) and the neuropsychological battery from the Consortium to Establish a Registry for Alzheimer's Disease (CERAD). ${ }^{11,12}$ The National Adult Reading Test (NART) was performed to rate premorbid IQ. ${ }^{13}$ Participants were considered to have mild cognitive impairment if they satisfied Petersen's original classification: ${ }^{14}$

(a) memory complaint, preferably corroborated by an informant;

(b) objective memory impairment;

(c) normal general cognitive function;

(d) intact activities of daily living;

(e) no diagnosis of dementia.

A diagnosis of mild cognitive impairment was made if the MMSE score was 25-27 and the participant's scores were 1.5 standard deviations below the published norms for any of the CERAD domains (relative to age, education and gender). ${ }^{15}$

\section{Diabetes mellitus}

Participants (and informants) were asked whether the participant had ever been diagnosed by a physician as having diabetes mellitus. They were also asked about all medications used on a daily basis. Antidiabetic medication use was validated by 
reviewing the general practitioners' prescription slips and medication boxes. Participants were classified as having diabetes mellitus if there was a report of physician diagnosis of the disorder with evidence of use of oral antidiabetic medications or insulin and information from the general practitioner. This was determined at baseline and every follow-up assessment.

\section{Other confounding factors}

History of hypertension, stroke, transient ischaemic attack (TIA) and coronary heart disease (myocardial infarction, angina and coronary artery bypass grafting) was ascertained at each assessment point. The medical history and medication use were further validated by reviewing the general practitioners' prescription slips and medication boxes. These too were determined at baseline and every follow-up assessment.

History of smoking (current and lifetime) and heavy alcohol drinking any time during lifetime was obtained from self-report. A shorter version of the Geriatric Depression Scale (GDS) was used for evaluating depressed mood, ${ }^{17}$ with a score above 5 being a potential indicator of current depression.

Venous blood was obtained for DNA extraction and genotyping for the apolipoprotein E (APOE) alleles using standard methods. ${ }^{16}$ The APOE haplotype was determined using two allelic discrimination assays (rs7412 and rs429358) based on fluorogenic 5 ' nuclease activity, the Taq polymerase single nucleotide polymorphism genotyping assay (TaqMan, Applied Biosystems Inc., www.appliedbiosystems.com).

\section{Diagnosis of dementia}

At follow-up a modified version of the Telephone Interview for Cognitive Status (TICS-M) $)^{18}$ and the GDS were administered over the telephone. If the TICS-M score was 22 or less, a full face-to-face assessment including evaluation of dementia was conducted. The assessment process has been described and fully referenced elsewhere, ${ }^{19}$ but in brief, participants and informants were interviewed using a structured interview including the Cambridge Examination for Mental Disorders of Older People (CAMDEX),${ }^{20}$ the MMSE for cognition, assessment of function using the Blessed Dementia Scale and the Bristol Activities of Daily Living Scale, ${ }^{21,22}$ the Neuropsychiatry Inventory for noncognitive symptoms, ${ }^{23}$ the Cornell Scale for Depression in Dementia, ${ }^{24}$ and the Webster Disability Scale for motor symptoms. ${ }^{25}$ Dementia syndromes were diagnosed using a validated algorithm and this assessment process has previously been shown to be highly accurate with respect to post-mortem diagnosis. $^{19}$

All participants gave informed consent. The study was approved by the joint Institute of Psychiatry and South London \& Maudsley National Health Service (NHS) Trust ethical committee.

\section{Statistical analysis}

Data were recorded and analysed with SPSS version 15.0 for Windows. The incidence rates of dementia per person-year were calculated as the number of new cases divided by the number of person-years at risk. Association of patient characteristics at baseline and conversion to dementia was tested using the chi-squared test and Student's $t$-test. The longitudinal data were analysed using Cox proportional hazard models both unadjusted and with additional adjustment for survival status. The backward stepwise method was used, which includes all the independent variables in a baseline regression model and successively removes the variables that contribute least to the model. In this way, a model is identified that provides the 'best fit' in terms of finding variables that are significant in predicting the dependent variable.

\section{Results}

Among the individuals identified from primary care practices who agreed to participate, 165 were considered to have possible mild cognitive impairment, of whom 103 fulfilled Peterson's criteria. The mean age of this cohort $(n=103)$ at baseline was 79.4 years (s.d. $=6.3$ ) and $66(64 \%)$ were women. They had 10.6 years of education (s.d. =2.6) with a mean baseline MMSE score of 26.3 (s.d.=1.1). Of the 95 participants who had APOE genotyping, 30 (32\%) had at least one APOE4 allele. Thirty-one (29\%) of the 92 participants assessed for mood with the GDS were positive for current depression.

The cohort was followed from 2001 through 2007. During this period 17 of the 103 participants (16\%) died, $6(6 \%)$ were lost to follow up, $3(3 \%)$ were not approached as they had moved too great a distance from the study site and $16(16 \%)$ refused to continue participating in the study. In total, 61 participants were successfully followed for 4 years (59\%). They were comparable in sociodemographic profile, risk factors and clinical parameters to those who did not complete follow up, except for being younger (mean 2 years). Sixteen (16\%) participants had a diagnosis of diabetes, with a mean duration of 9.3 years (s.d. $=5.4$ ). Among the 61 participants who successfully completed the follow-up for 4 years there were 19 (31\%) incident cases of dementia, 2 (3\%) participants improved in their cognition, reverting to normal cognitive levels, and $40(59 \%)$ remained stable. The mean annual conversion rate to dementia was $8 \%$. The dementia diagnoses were predominantly Alzheimer's disease (84\%; probable, $n=11$, possible, $n=5$ ); mixed Alzheimer's disease and vascular dementia $(5 \% ; n=1)$; vascular dementia $(5 \% ; n=1)$; and dementia of Lewy body type $(5 \% ; n=1)$.

The baseline characteristics of participants with and without diabetes mellitus are compared in Table 1. Compared with participants without diabetes, those with the disorder had a greater prevalence of current depression, a history of heavy drinking, and increased rates of stroke and/or TIA and coronary heart disease. Table 2 shows the baseline characteristics of the participants who progressed to develop dementia compared with those who did not. Those who converted to dementia were older, had a greater prevalence of diabetes, a longer duration of diabetes and a higher prevalence of stroke and/or TIA. Among those who converted to dementia, 7 participants had diabetes, of whom 6 developed Alzheimer's disease.

All the variables were further analysed using Cox proportional regression models. Both unadjusted and adjusted models (Table 3) identified diabetes as the only significant predictor. The backward stepwise method showed presence of diabetes as a significant predictor for conversion to dementia, with a hazard ratio of 2.9 (95\% CI 1.1-7.3).

Seven participants showed deterioration in cognition during follow-up on TICS-M and MMSE, but did not wish to have a complete assessment towards a formal dementia diagnosis. Although these individuals were likely to be converters they were excluded from the analysis. Including them in the analysis gave a group size of $n=26$ for participants showing deterioration, and Cox regression analysis models demonstrated that diabetes continued to be the only variable associated with increased risk of progression to dementia (hazard ratio 2.5, 95\% CI 1.1-5.7, $P=0.03)$. The two participants who reverted to normal cognition did not have diabetes, were 82.5 (s.d. $=3.6$ ) years old, had a mean MMSE score of 26.5 (s.d.=0.7) and had 12.5 (s.d.=4.9) years of education. They continued to have normal cognition during the remaining period of the study. 
Table 1 Comparison of sociodemographic variables, cognitive parameters and medical history of participants with mild cognitive impairment with or without diabetes

\begin{tabular}{|c|c|c|c|}
\hline & $\begin{array}{l}\mathrm{MCl} \text { with } \\
\text { diabetes } \\
(n=16)\end{array}$ & $\begin{array}{l}\mathrm{MCl} \text { without } \\
\text { diabetes } \\
(n=87)\end{array}$ & $P$ \\
\hline Gender: female, $n$ (\%) & $7(44)$ & $59(68)$ & $0.065^{\mathrm{a}}$ \\
\hline Age, years: mean (s.d.) & $79(7.5)$ & $79.5(6.1)$ & $0.79^{\mathrm{b}}$ \\
\hline \multicolumn{4}{|l|}{ Outcome in 4 years, $n(\%)$} \\
\hline Death & $4(25)$ & $13(15)$ & \\
\hline Conversion to dementia & $7(44)$ & $12(14)$ & \\
\hline Loss to follow-up & $2(12)$ & $23(26)$ & \\
\hline Reversion to normal cognition & $0(0)$ & $2(2)$ & \\
\hline $\mathrm{MCl}$ stable & $3(19)$ & $37(42)$ & $0.016^{a}$ \\
\hline Education, years: mean (s.d.) & $10.3(1.4)$ & $10.7(2.8)$ & $0.63^{b}$ \\
\hline Premorbid NART score: mean (s.d.) & $24.7(8.7)$ & $21(9.7)$ & $0.25^{\mathrm{b}}$ \\
\hline MMSE score: mean (s.d.) & $26.8(0.8)$ & $26.3(1.1)$ & $0.06^{\mathrm{b}}$ \\
\hline Current depression, $n(\%)^{c, d}$ & $8(42)$ & $23(30)$ & $0.04^{\mathrm{a}}$ \\
\hline APOE4 genotype, $n(\%)^{\mathrm{e}, \mathrm{f}}$ & $4(25)$ & $26(33)$ & $0.76^{\mathrm{a}}$ \\
\hline History of heavy drinking, $n$ (\%) & $4(25)$ & $1(1)$ & $<0.001^{\mathrm{a}}$ \\
\hline \multicolumn{4}{|l|}{ Cigarette smoking, $n$ (\%) } \\
\hline Current & $4(25)$ & $14(16)$ & \\
\hline Lifetime & $6(38)$ & $25(29)$ & $0.46^{\mathrm{a}}$ \\
\hline Hypertension, $n$ (\%) & $8(50)$ & $44(51)$ & $0.91^{\mathrm{a}}$ \\
\hline Stroke/TIA, $n(\%)$ & $3(19)$ & $4(5)$ & $0.03^{\mathrm{a}}$ \\
\hline Coronary heart disease, $n$ (\%) & $9(56)$ & 27 (31) & $0.052^{\mathrm{a}}$ \\
\hline \multicolumn{4}{|c|}{$\begin{array}{l}\text { MCI, mild cognitive impairment; MMSE, Mini-Mental State Examination; NART, } \\
\text { National Adult Reading Test; TIA, transient ischaemic attack. } \\
\text { a. Calculated using the } \chi^{2} \text {-test. } \\
\text { b. Calculated using Student's } t \text {-test. } \\
\text { c. Geriatric Depression Score: cut-off score }>5 \text {. } \\
\text { d. Eleven missing values. } \\
\text { e. Presence of at least one } \varepsilon 4 \text { allele. } \\
\text { f. Eight missing values. }\end{array}$} \\
\hline
\end{tabular}

Table 2 Comparison of sociodemographic variables, cognitive parameters and medical history of participants with mild cognitive impairment categorised by conversion to dementia

\begin{tabular}{|c|c|c|c|}
\hline & $\begin{array}{l}\text { Conversion } \\
\text { to dementia } \\
\qquad(n=19)\end{array}$ & $\begin{array}{l}\text { Non- } \\
\text { conversion } \\
(n=40)\end{array}$ & $P$ \\
\hline Gender: female, $n$ (\%) & $15(79)$ & $24(60)$ & $0.15^{\mathrm{a}}$ \\
\hline Age, years: mean (s.d.) & $79.5(5.5)$ & $77.3(5.7)$ & $0.2^{\mathrm{b}}$ \\
\hline Diabetes, $n(\%)$ & $7(37)$ & $3(8)$ & $0.005^{a}$ \\
\hline Education, years: mean (s.d.) & $10.9(1.9)$ & $10.4(2.9)$ & $0.54^{\mathrm{b}}$ \\
\hline Premorbid NART score: mean (s.d.) & $23.1(10.6)$ & $19.9(8.7)$ & $0.29^{b}$ \\
\hline MMSE score: mean (s.d.) & $26.4(1.2)$ & $26.3(1.2)$ & $0.67^{\mathrm{b}}$ \\
\hline Current depression, $n(\%)^{c, d}$ & $6(38)$ & $9(26)$ & $0.39^{a}$ \\
\hline APOE4 genotyping, $n(\%)^{e, f}$ & $8(36)$ & $8(22)$ & $0.15^{a}$ \\
\hline History of heavy drinking, $n(\%)$ & $3(16)$ & $1(2)$ & $0.06^{\mathrm{a}}$ \\
\hline \multicolumn{4}{|l|}{ Cigarette smoking, $n$ (\%) } \\
\hline Current & $2(10)$ & $6(15)$ & $0.78^{a}$ \\
\hline Lifetime & $5(26)$ & $12 \underline{(31)}$ & \\
\hline Diabetes duration, years: mean (s.d.) & $3.1(4.8)$ & $0.7(3.0)$ & $0.027^{b}$ \\
\hline Hypertension, $n$ (\%) & $8(42)$ & $22(55)$ & $0.26^{a}$ \\
\hline Stroke/TIA, $n(\%)$ & $4(21)$ & $0(0)$ & $0.003^{\mathrm{a}}$ \\
\hline Coronary heart disease, $n$ (\%) & $7(37)$ & $16(40)$ & $0.82^{\mathrm{a}}$ \\
\hline \multicolumn{4}{|c|}{$\begin{array}{l}\text { MMSE, Mini-Mental State Examination; NART, National Adult Reading Test; } \\
\text { TIA, transient ischaemic attack. } \\
\text { a. Calculated using the } \chi^{2} \text {-test. } \\
\text { b. Calculated using Student's } t \text {-test. } \\
\text { c. Geriatric Depression Score: cut-off score }>5 \text {. } \\
\text { d. Eight missing values. } \\
\text { e. Presence of at least one } \varepsilon 4 \text { allele. } \\
\text { f. Six missing values. }\end{array}$} \\
\hline
\end{tabular}

\section{Discussion}

This prospective longitudinal study shows that diabetes is associated with a substantially increased risk of conversion of mild cognitive impairment to dementia. Our finding supports the hypothesis that diabetes is linked to cognitive dysfunction.

\section{Incidence of dementia}

The rate of progression to dementia in our study (8\%) was slightly lower than but broadly consistent with previous research. ${ }^{2,26,27}$ The most common diagnosis post-conversion was Alzheimer's disease $(n=16,84 \%)$ and one person had a diagnosis of mixed Alzheimer's disease with vascular dementia. As in other studies, the conversion rates showed a high probability of receiving a diagnosis of Alzheimer's disease. ${ }^{26,27}$

\section{Diabetes, other confounding factors and risk of conversion}

Previous longitudinal studies of the risk of dementia in elderly people with diabetes have shown a consistent pattern with a hazard ratio of 1.5, with an overall increase in incidence of $50-100 \%$ relative to people without diabetes. ${ }^{5}$ Diabetes was associated with a higher risk of incident mild cognitive impairment in a population with a high prevalence of this disorder $\left(\mathrm{HR}=1.4,95 \%\right.$ CI 1.1-1.8). ${ }^{4}$ Both cognitive impairment and diabetes may be important independent risk factors for dementia, which, when combined, confer even greater risk, as reflected in the almost 3 times greater potential hazard of developing any type of dementia $(\mathrm{HR}=2.9,95 \%$ CI 1.1-7.3) in our study. The risk remained consistent even after adjustment for gender, age, vascular risk factors such as stroke and/or TIA and coronary heart disease, and diabetes-specific features such as diabetes duration, which are important determinants for increased risk of dementia in people with diabetes. ${ }^{7}$

The prevalence of diabetes in our sample was consistent with the reports for a White population aged around 70 years, i.e. 10$20 \% .^{6}$ Longer duration of diabetes has been associated with an increased risk of developing mild cognitive impairment. ${ }^{28}$ The duration of diabetes was longer in members of our cohort who progressed to dementia, although this did not show an association when adjusted for other variables (see Table 3). A possible explanation could be the small number of participants. In our study, individuals with diabetes had greater prevalence of cardiovascular and cerebrovascular disease, i.e. stroke, TIA, angina, myocardial infarction and coronary artery bypass grafting. This is consistent with reports that people with diabetes have increased vascular risk factors, and it has been suggested that it is this that increases the risk of dementia. ${ }^{29}$ However, although stroke or TIA was associated with increased risk of progression to dementia, this association did not remain when adjusted for presence and duration of diabetes, age and gender (see Table 3).

Apolipoprotein E genotype did not have an effect on the risk of conversion to dementia or Alzheimer's disease from cognitive impairment in our study. This was in accordance with a recent study which reported that carrying at least one APOE4 allele was a risk factor for the development of mild cognitive impairment, but that once impairment was established the gene had no effect on the risk of progressing to Alzheimer's disease. ${ }^{30}$

\section{Limitations}

The main limitation of this study was that we did not have measures of glycaemia to ascertain undiagnosed diabetes or to 
Table 3 Hazard ratios for unadjusted and adjusted analyses

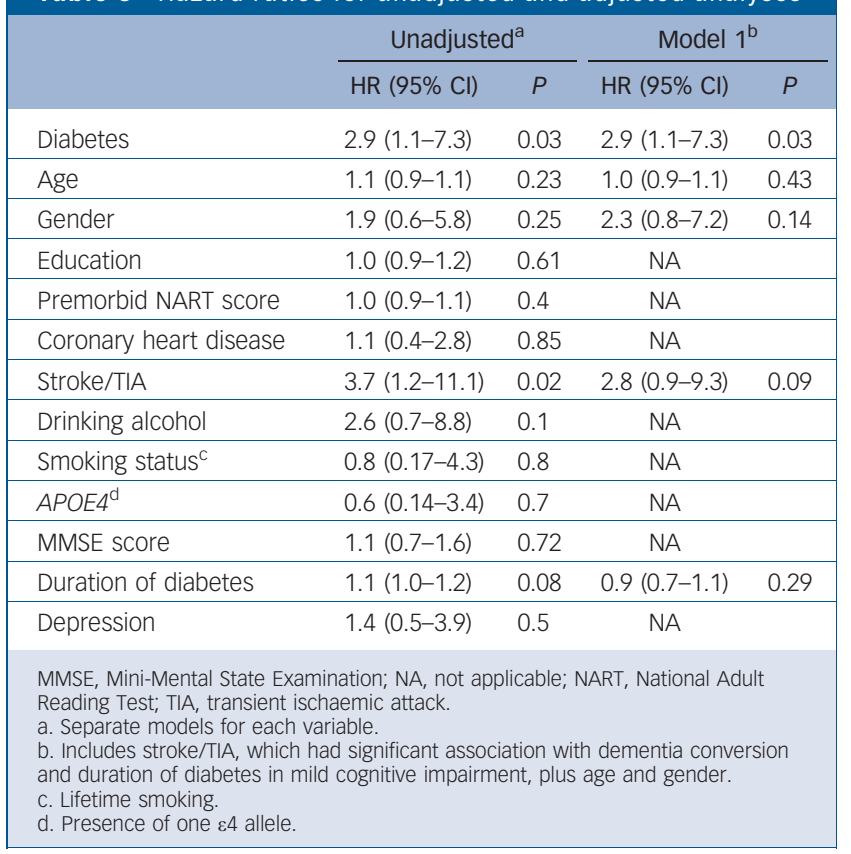

determine the degree of diabetic control. This almost certainly leads to an underestimate of the prevalence of diabetes. However, any such underestimate is unlikely to alter the conclusions substantially because of the longitudinal study design. If anything, an underestimate of underlying diabetes might act to reduce the size of the effect on conversion. Although our sample number was modest, recruitment from primary practices rather than memory clinics increases the representativeness of the study population. Our finding of a relatively low rate of conversion from mild cognitive impairment reflects this recruitment strategy and is in line with other studies with sample populations that approximate more closely to the general population than those drawn from secondary care specialist clinics. $^{2}$ Our cohort consisted of White European participants, and it would be interesting to repeat this study in different populations, as the prevalence of diabetes is higher in some ethnic groups. ${ }^{4}$

\section{Potential mechanisms and implications}

Diabetes might accelerate cognitive decline and conversion to dementia by a number of potential mechanisms. These include insulin resistance syndrome, disturbances in insulin homeostasis in the brain, hyperinsulinaemia, interplay with the insulin degrading enzyme involved in both insulin and amyloid proteolysis or effects of insulin signalling on tau metabolism and generation of advanced products of glycosylation. ${ }^{8}$ Whatever the mechanism, with an expected increase in prevalence of diabetes in people of all ages including older adults, ${ }^{8}$ the risk of developing dementia may increase. Identification of those at particular risk of progression might help to target early treatment - both pharmacological and social.

There are few data to guide evidence-based decision-making for patients with diabetes and cognitive impairment. Our study demonstrates that individuals with mild cognitive impairment and diabetes are at increased risk of developing dementia. This suggests the need for studies of improved diabetes control and related approaches as possible strategies for early intervention.
Latha Velayudhan, DNB(Psychiatry), National Institute for Health Research (NIHR) Biomedical Research Centre for Mental Health, and Medical Research Council (MRC) Centre for Neurodegeneration Research, Institute of Psychiatry; Michaela Poppe, PhD, Nicola Archer, PhD, Section of Old Age Psychiatry, Institute of Psychiatry; Petroula Proitsi, PhD, NIHR Biomedical Research Centre for Mental Health and MRC Centre for Neurodegeneration Research, Institute of Psychiatry; Richard G. Brown, Centre for Neurodegeneration Research, Institute of Psychiatry; Richard G. Brow
PhD, NIHR Biomedical Research Centre for Mental Health and MRC Centre for Neurodegeneration Research, Institute of Psychiatry, and Department of Psychology, King's College London; Simon Lovestone, PhD, NIHR Biomedical Research Centre and MRC Centre for Neurodegeneration Research, Institute of Psychiatry, London, UK

Correspondence: Dr Latha Velayudhan, PO Box 70, Section of Old Age Psychiatry, Institute of Psychiatry, De Crespigny Park, London SE5 8AF, UK. E-mail: Latha.Velayudhan@kcl.ac.uk

First received 3 Mar 2009, final revision 1 Sep 2009, accepted 26 Sep 2009

\section{Funding}

The study was funded by the Alzheimer's Research Trust and the UK Medical Research Council, and through the National Institute for Health Research Specialist Biomedical Research Centre for Mental Health at the South London \& Maudsley National Health Service Foundation Trust.

\section{Acknowledgements}

We particularly thank Lee Wilding, research administrator, Section of Old Age Psychiatry, Institute of Psychiatry, London, for carrying out TICS-M telephone screening interviews Institute of Psychiatry, London, for carrying out
for the majority of participants in the community.

\section{References}

1 Ferri CP, Prince M, Brayne C, Brodaty H, Fratiglioni L, Ganguli M, et al. Global prevalence of dementia: a Delphi consensus study. Lancet 2005; 366: 2112-7.

2 Bruscoli M, Lovestone S. Is MCI really just early dementia? A systematic review of conversion studies. Int Psychogeriatr 2004; 16: 129-40.

3 Yaffe K, Blackwell T, Kanaya AM, Davidowitz N, Barrett-Connor E, Krueger K Diabetes, impaired fasting glucose, and development of cognitive impairment in older women. Neurology 2004; 63: 658-63.

4 Luchsinger JA, Reitz C, Patel B, Tang MX, Manly JJ, Mayeux R. Relation of diabetes to mild cognitive impairment. Arch Neurol 2007; 64: 570-5.

5 Biessels GJ, Staekenborg S, Brunner E, Brayne C, Scheltens P. Risk of dementia in diabetes mellitus: a systematic review. Lancet Neurol 2006 5: $64-74$

6 Biessels GJ, Deary IJ, Ryan CM. Cognition and diabetes: a lifespan perspective. Lancet Neurol 2008; 7: 184-90.

7 Das SK, Bose P, Biswas A, Dutt A, Banerjee TK, Hazra AM, et al. An epidemiologic study of mild cognitive impairment in Kolkata, India. Neurology 2007; 68: 2019-26.

8 Zimmet P, Alberti KG, Shaw J. Global and societal implications of the diabetes epidemic. Nature 2001; 414: 782-7.

9 Ellul J, Archer N, Foy CM, Poppe M, Boothby H, Nicholas H, et al. The effects of commonly prescribed drugs in patients with Alzheimer's disease on the rate of deterioration. J Neurol Neurosurg Psychiatry 2007; 78: 233-9.

10 Hye A, Lynham S, Thambisetty M, Causevic M, Campbell J, Byers $\mathrm{HL}$, et al. Proteome-based plasma biomarkers for Alzheimer's disease. Brain 2006; 129: $3042-50$

11 Folstein MF, Folstein SE, McHugh PR. 'Mini-mental state': a practical method for grading the cognitive state of patients for the clinician. J Psychiatr Res 1975; 12: 189-98.

12 Morris JC, Mohs RC, Rogers H, Fillenbaum G, Heyman A. Consortium to Establish a Registry for Alzheimer's Disease (CERAD) Clinical and neuropsychological assessment of Alzheimer's disease. Psychopharmacol Bull 1988; 24: 641-52.

13 Nelson HE. National Adult Reading Test. Test Manual. NferNelson, 1982.

14 Petersen RC, Smith GE, Waring SC, Ivnik RJ, Tangalos EG, Kokmen E. Mild cognitive impairment: clinical characterization and outcome. Arch Neurol 1999; 56: 303-8.

15 Lopez OL, Jagust WJ, DeKosky ST, Becker JT, Fitzpatrick A, Dulberg C, et al. Prevalence and classification of mild cognitive impairment in the cardiovascular health study cognition study. Arch Neurol 2003; 60: 1385-9.

16 Wenham PR, Price WH, Blandell G. Apolipoprotein E genotyping by one-stage PCR. Lancet 1991; 337: 1158-9. 
17 Yesavage JA, Brink TL, Rose TL, Lum O, Huang V, Adey M, et al. Development and validation of a geriatric depression screening scale: a preliminary report. J Psychiatr Res 1983; 17: 37-49.

18 De Jager CA, Budge MM, Clarke R. Utility of TICS-M for the assessment of cognitive function in older adults. Int J Geriatr Psychiatry 2003; 18: 318-24.

19 Foy CM, Nicholas $\mathrm{H}$, Hollingworth $\mathrm{P}$, Boothby $\mathrm{H}$, Williams J, Brown RG, et al. Diagnosing Alzheimer's disease - non-clinicians and computerised algorithms together are as accurate as the best clinical practice. Int J Geriatr Psychiatry 2007; 22: 1154-63.

20 Roth M, Tym E, Mountjoy CQ, Huppert FA, Hendrie H, Verma S, Goddard R CAMDEX. A standardised instrument for the diagnosis of mental disorder in the elderly with special reference to the early detection of dementia. Br J Psychiatry 1986; 149: 698-709.

21 Blessed G, Tomlinson BE, Roth $M$. The association between quantitative measures of dementia and of senile change in the cerebral grey matter of elderly subjects. Br J Psychiatry 1968; 114: 797-811.

22 Bucks RS, Ashworth DL, Wilcock GK, Siegfried K. Assessment of activities of daily living in dementia: development of the Bristol Activities of Daily Living Scale. Age Ageing 1996; 25: 113-20.

23 Cummings JL, Mega M, Gray K, Rosenberg-Thompson S, Carusi DA, Gornbein J. The Neuropsychiatric Inventory: comprehensive assessment of psychopathology in dementia. Neurology 1994; 44: 2308-14.
24 Alexopoulos GS, Abrams RC, Young RC, Shamoian CA. Cornell Scale for Depression in Dementia. Biol Psychiatry 1988; 23: 271-84.

25 Webster DD. Critical analysis of the disability in Parkinson's disease. Mod Treat 1968; 5: 257-82.

26 Fischer $\mathrm{P}$, Jungwirth $\mathrm{S}$, Zehetmayer $\mathrm{S}$, Weissgram $\mathrm{S}$, Hoenigschnabl S, Gelpi $\mathrm{E}$, et al. Conversion from subtypes of mild cognitive impairment to Alzheimer dementia. Neurology 2007; 68: 288-91.

27 Yaffe K, Petersen RC, Lindquist K, Kramer J, Miller B. Subtype of mild cognitive impairment and progression to dementia and death. Dement Geriatr Cogn Disord 2006; 22: 312-9.

28 Roberts RO, Geda YE, Knopman DS, Christianson TJ, Pankratz VS, Boeve BF, et al. Association of duration and severity of diabetes mellitus with mild cognitive impairment. Arch Neurol 2008; 65: 1066-73.

29 Fillit $\mathrm{H}$, Nash DT, Rundek T, Zuckerman A. Cardiovascular risk factors and dementia. Am J Geriatr Pharmacother 2008; 6: 100-18.

30 Barabash A, Marcos A, Ancín I, Vázquez-Alvarez B, de Ugarte C, Gil P, et al. APOE, ACT and CHRNA7 genes in the conversion from amnestic mild cognitive impairment to Alzheimer's disease. Neurobiol Aging 2009; 30: 1254-64.

\section{Jean-Pierre Schuster and Yann Le Strat}

The name of Désiré Bourneville is linked with tuberous sclerosis complex, which he documented and named. He also contributed to major hospital reforms, especially in the care of children with intellectual disabilities. His medical and social work with such children made him a precursor in child and adolescent psychiatry.

Bourneville was born in 1840 in Garencières, a small village in Normandy. In 1865 he became resident at the Paris Medical Faculty and 5 years later submitted his doctoral thesis on clinical thermometry in brain diseases. When working at Salpêtriere Hospital, he played a major role in the publication of the Lessons of Charcot, his mentor, and in the studies on hysteria about which he published an important photographic series in collaboration with Regnard. He was a prolific editor of several journals. In 1873 he founded the Progrès Médical, a popular and influential journal that helped in the dissemination of medical science and hygiene.

He developed a consistent hospital policy of secularisation, founded the first nurse school in France and contributed to regulating midwifery in Paris. (Between 1877 and 1881, Bourneville wrote several reports to the authorities in favour of organising separate hospital departments for pregnant women. In part because of his advocacy, a new order of medical practitioners was created for obstetricians in Paris.) Throughout his life, he was devoted to training caregivers and improving their living standards. As a medical reformist, he was interested in politics and was a councillor in Paris (1876) and an MP (1883-1889).

In 1879, Bourneville was appointed as Head of the Psychiatry Department of Bicêtre Hospital, taking care of 'epileptics and idiots children'. He was a defender of out-patient treatments, breaking with a tradition of confinement. His major achievement was to transform the psychiatric unit into a 'school asylum', foreshadowing a medico-pedagogical institute. Bourneville promoted a therapeutic and educational approach, inspired by the work of Seguin, and involved medical and public authorities in his work towards the integration of children with intellectual disability into society. 